\title{
Validación de un Instrumento sobre Metacognición para Estudiantes de Segundo Ciclo de Educación General Básica*
}

\author{
Validation of an Instrument on Metacognition for Second Cycle \\ General Basic School Pupils \\ Validação de um instrumento de metacognição para estudantes do segundo ciclo de \\ Educação Geral básica
}

Sandra Jaramillo, ${ }^{\text {a Sonia Osses }}{ }^{\mathrm{a}}$

aUniversidad de La Frontera, Facultad de Educación y Humanidades, Departamento de Educación. Avda. Francisco Salazar 01145, Temuco. Correo electrónico: sjaramillo_7@hotmail.com, sosses@ufro.cl

\section{RESUMEN}

El presente artículo da cuenta de la validación de un Instrumento diseñado para medir metacognición en términos de: conocimiento, experiencias metacognitivas y autorregulación cognitiva. El instrumento, conformado por 33 ítems tipo Escala de Likert, está dirigido a estudiantes de $7^{\circ}$ y $8^{\circ}$ Año Básico de Escuelas Municipalizadas de alta vulnerabilidad de la Región de La Araucanía, Chile. Se evaluó: validez de contenido por juicio de expertos, validez de constructo mediante Análisis Factorial y confiabilidad por medio del Alpha de Cronbach. El juicio de expertos permitió contrastar cada uno de los ítems con la teoría sobre metacognición; el análisis factorial, agrupar los ítems en torno a los factores que componen dicho constructo. El valor de Alfa obtenido $(0,860)$ corresponde a muy alto lo que significa que el instrumento puede ser utilizado como técnica confiable de recolección de datos.

Palabras clave: metacognición, validez de contenido, validez de constructo, confiabilidad.

\begin{abstract}
This article reports the validation of an Instrument designed for measuring metacognition in terms of knowledge, metacognition experiences and cognitive auto-regulation. The instrument, consisting of 33 Likert Scale type items, is addressed to pupils of 7th and 8th Basic Grades of high-risk Municipalized Schools in the Araucanía Region, Chile. Content validity was evaluated by experts' judgment, construct validity by means of Factorial Analysis and reliability by means of Cronbach's Alpha. Experts' judgment allowed the contrast of every item through metacognition theory, and factorial analysis grouping the items around the factors constituting the construct. The obtained Alfa value (0.860) is very high (Ruiz, 1998) meaning that the instrument can be used as a reliable technique of data collection.

Key words: metacognition, content validity, construct validity, reliability.
\end{abstract}

\section{RESUMO}

Informa-se sobre validação de um Instrumento criado para medir metacognição em termos de: conhecimento, experiências metacognitivas e autorregulação cognitiva. O instrumento, formado por 33 itens em Escala de Likert, está dirigido a estudantes de $7^{\circ}$ e $8^{\circ}$ ano básico de escolas municipalizadas, de alta vulnerabilidade da região de La Araucanía, Chile. Avaliou-se: validade de conteúdo por peritos, validade de constructo mediante Análise Fatorial e confiabilidade por meio do Alfa de Cronbach. O julgamento de peritos permitiu contrastar cada um dos itens com a teoria sobre metacognição; a análise fatorial agrupa os itens em torno dos fatores que compõem o constructo. O valor de Alfa obtido (0,860) corresponde a muito alto (Ruiz, 1998), o que significa que o instrumento pode ser utilizado como técnica de correlação de dados.

Palavras chave: metacognición, validade de conteúdo, validade do constructo, confiabilidade.

* $\quad$ Artículo elaborado en el marco del Proyecto Fondecyt 1100378: "Hacia un aprendizaje de calidad en la educación científica. Estrategias didácticas para el desarrollo de capacidades y valores” 


\section{INTRODUCCIÓN}

La Educación del presente siglo requiere de la búsqueda de metodologías para posibilitar que los alumnos, interactuando con sus pares, sean los protagonistas en la construcción y apropiación del conocimiento y, por consiguiente, desarrollen grados crecientes de autonomía. La autonomía del aprendizaje, basada en la conciencia que cada estudiante posee acerca de los propios procesos cognitivos y su regulación, constituye un concepto clave en términos del desarrollo de la facultad de tomar decisiones en función de un objetivo. Esta capacidad del ser humano se denomina Metacognición.

Por el alto valor formativo de este constructo en educación, es que adquiere gran importancia diseñar instrumentos válidos y confiables que permitan cuantificar el nivel de metacognición alcanzado por los estudiantes y, eventualmente, el desarrollo metacognitivo logrado por ellos en un lapso de tiempo determinado.

En coherencia con las afirmaciones anteriores, en este artículo se presenta el proceso de validación de un instrumento orientado a medir el nivel de metacognición en alumnos de $7^{\circ}$ y $8^{\circ}$ Año de la Escuela Básica Municipalizada Metrenco -cuyos estudiantes presentan una alta vulnerabilidad social- ubicada en la Comuna de Padre Las Casas, Región de la Araucanía, Chile.

\section{MARCO REFERENCIAL}

La metacognición es una de las áreas de investigación que más ha contribuido a la configuración de las nuevas concepciones del aprendizaje y de la instrucción. A medida que se han ido imponiendo las concepciones constructivistas del desarrollo y del aprendizaje, se ha ido atribuyendo un papel creciente a la conciencia que tiene el sujeto y a la regulación que ejerce sobre su propio aprendizaje (Carretero, 2001:12).

Según Flavell (Cit. en Mateos, 2001: 21-22), "la metacognición se refiere al conocimiento que uno tiene de sus propios procesos cognitivos y a la autorregulación de estos procesos con relación a alguna meta". En otras palabras, el autor identifica a la metacognición con el conocimiento de la actividad cognitiva y con el control que se puede ejercer sobre ella.

Soto (2002) coincide con esta concepción al afirmar que la metacognición está asociada a dos componentes: a) el conocimiento metacognitivo, que involucra acciones referidas a las personas, tareas y estrategias y b) la regulación de los procesos cognitivos, que incluyen su planificación, supervisión y evaluación, procesos que van más allá de lo cognitivo, puesto que se refieren a un plano reflexivo sobre lo cognitivo.

El primer tipo de conocimiento metacognitivo comprende el conocimiento y las creencias en relación a las características de las personas consigo mismas, en cuanto a las propias habilidades, recursos y experiencias en la realización de diversas tareas cognitivas, intereses, motivaciones y estados personales que pueden afectar el rendimiento; en relación con otras personas, por ejemplo, saber que a una le resulta más fácil una tarea que a otra y, en relación con el ser humano como ser cognitivo, como saber que en una situación de aprendizaje respecto de una imagen, en primer lugar se percibe la totalidad y, luego, los detalles. 
El segundo tipo de conocimiento metacognitivo se refiere al conocimiento acerca de la influencia de la naturaleza de la tarea sobre la facilidad o dificultad para llevarla a cabo.

El tercer tipo de conocimiento metacognitivo se refiere a la efectividad del uso de diferentes estrategias para desarrollar exitosamente una tarea. Por ejemplo, saber que, si se pretende comprender en profundidad un proceso natural, como la fotosíntesis, se logrará mejores resultados comenzando con una actividad de laboratorio que leyendo repetidas veces un documento que aborde el tema desde la perspectiva teórica.

De acuerdo a lo anterior, los conocimientos metacognitivos están conformados por datos relativos a la persona, la tarea y las estrategias y, además, por las experiencias metacognitivas, referidas a ideas, pensamientos, sensaciones o sentimientos que acompañan a la acción cognitiva y pueden culminar en su autorregulación.

Según Argüelles (2007), la regulación de los procesos cognitivos se refiere a los aspectos: planificación, supervisión y evaluación, que se ponen en marcha a objeto de asegurar el logro de las metas a través del control de la actividad cognitiva, de modo tal que se generen procesos de aprendizaje significativos.

Durante la planificación, se diseñan y proyectan las acciones a ejecutar, culminando con la elaboración de un plan de acción. Esta etapa es fundamental para la correcta realización de la fase siguiente: la supervisión.

En la etapa de supervisión, se revisa la evolución de la actividad cognitiva, se verifican los avances y se toman medidas correctivas orientadas a dirigir los esfuerzos hacia el cumplimiento de los objetivos.

La evaluación está dirigida a comprobar las metas logradas y a identificar los problemas suscitados durante el proceso a objeto de generar nuevas acciones o utilizar nuevas estrategias que hagan posible el logro de las metas establecidas (Argüelles, 2007).

En resumen, es posible diferenciar dos componentes metacognitivos, uno de naturaleza declarativa y otro de carácter procedimental, ambos importantes para el aprendizaje e interrelacionados, de modo tal que el alumno aventajado emplea sus conocimientos metacognitivos para autorregular eficazmente su aprendizaje y, a su vez, dicha regulación puede conducirle a lograr nuevos conocimientos.

\subsection{CÓMO DESARROLLAR HABILIDADES METACOGNITIVAS}

Según Bermeosolo (2005:213), "El objetivo del desarrollo de las habilidades metacognitivas, las que pueden ser enseñadas de una manera explícita, es convertir al estudiante o a cualquier persona en un usuario hábil de su propio conocimiento". Esto significa que el desarrollo de tales habilidades permite al estudiante organizar su potencial de aprendizaje de manera que lo pueda adaptar a diferentes contextos; en otras palabras, le facilita el camino hacia su aprendizaje autónomo, aprendiendo a aprender.

Para desarrollar habilidades metacognitivas, es necesario considerar aspectos tales como: la naturaleza de la metacognición y la edad de los estudiantes, entre otros. Al respecto, Mateos (2001), por una parte, señala que el conocimiento metacognitivo surge tarde en el desarrollo del sujeto, en cambio, la regulación metacognitiva, depende menos de la edad. Al respecto, afirma: "Algunos aspectos del conocimiento de las estrategias, concretamente los aspectos condicionales, no parecen adquirirse antes de la adolescencia. Muchos niños de doce años no parecen ser conscientes aún de la necesidad de desplegar un comportamiento estratégico diferente para ajustarse a las demandas de la tarea" (Mateos, 2001: 60). 
A partir de estos antecedentes, se infiere que el desarrollo de habilidades metacognitivas debiera ser considerado en el currículum escolar chileno a partir de $7^{\circ}$ Año Básico, curso en que la edad de los estudiantes, en general, fluctúa entre 12 y 14 años.

En cuanto a las estrategias de aprendizaje metacognitivas, Monereo et al. (2001) plantean que pueden y deben enseñarse conjuntamente con las distintas disciplinas que conforman el currículum, sin añadir tiempo adicional y con los recursos que posee cualquier docente. De esta afirmación, se desprende que dichos autores toman partido respecto de la modalidad en que se debe llevar a cabo el proceso de metacognición, optando por considerar el desarrollo metacognitivo como un medio para aprender y no como un fin en sí mismo.

Bermeosolo (2005) señala que, el docente para lograr un avance en la metacognición, deberá desarrollar explícitamente en los estudiantes habilidades, tales como: planificación, predicción, verificación con la realidad, autorregulación, control, comprobación de resultados y evaluación. Otro camino para lograrlo, sería invitar al estudiante a plantearse preguntas y autoinstrucciones, como las siguientes: ¿Tengo claro lo que debo aprender? ¿Sé lo que debo hacer para lograrlo? ¿Poseo la información necesaria para empezar a trabajar? Si no es así ¿sé cómo obtenerla? ¡Haz un alto en el camino para revisar lo que has hecho hasta el momento!

Además de los elementos considerados, es necesario tener presente que, para desarrollar la metacognición en términos de conocimiento y autorregulación, es fundamental contar con la motivación del estudiante. Al respecto, Mateos (2001:46) señala: "para que ese conocimiento de las estrategias cognitivas y metacognitivas se transforme en acción, tiene que ir acompañado de las intenciones o metas apropiadas y de un patrón de creencias positivas sobre los propios recursos para llevarlas a cabo".

\subsection{CÓMO EVALUAR LA METACOGNICIÓN}

Según Jiménez (2004), evaluar la metacognición no es medir cuánto dice o hace un sujeto, sino ayudarle a tomar conciencia de las estrategias que utiliza durante la ejecución de una tarea.

Pese a la necesidad de desarrollar habilidades metacognitivas durante el proceso de formación de los estudiantes en Educación General Básica, en este nivel en Chile y, probablemente, sólo existen algunas investigaciones respecto del desarrollo de habilidades metacognitivas referidas a comprensión de textos escritos (Peronard et al., 2000).

Considerando lo anterior, y en orden a la elaboración de instrumentos de recolección de datos para la investigación en curso: "Procesos Metacognitivos en el Currículum de Ciencias Naturales a nivel de Educación General Básica", ha sido necesario construir un instrumento confiable y con un grado de validez aceptable, que mida metacognición en términos de autoconocimiento y autorregulación -tema central del presente artículodirigido a estudiantes en condiciones de alta vulnerabilidad social, de Séptimo y Octavo Año Básico de la Región de La Araucanía, Chile. 


\section{ELABORACION Y VALIDACIÓN DEL INSTRUMENTO}

\subsection{ELABORACIÓN DEL INSTRUMENTO}

Como señala Jiménez (2004: 64), "la metacognición, como constructo complejo, cuya conducta en los sujetos no es observable, es preciso medirla indirectamente". Para este efecto, se elaboró una Escala tipo Likert basada en: a) un instrumento validado con adolescentes chilenos de Liceos Municipalizados de alta vulnerabilidad (Osses, 2008, 2011) y b) en el protocolo propuesto por Colás y Buendía (1998) orientado a: definir la variable a medir, que en la presente investigación corresponde al nivel de desarrollo de la metacognición de los estudiantes sobre la base de los componentes del conocimiento metacognitivo y su autorregulación; formular los ítems o proposiciones acerca de la variable; seleccionar los ítems que más interesan, eliminando los ambiguos, los que contienen una doble opinión y los que no miden lo mismo que los demás.

En cuanto a las alternativas de respuesta presentes en la escala, Canales (2006) sugiere un mínimo de 3 y un máximo de 7 . Sin embargo, al respecto, se debe considerar factores como la edad y nivel educacional de los sujetos. Esto significa que es pertinente incluir un número reducido de opciones de respuesta en la escala a validar, dirigida a los estudiantes de $7^{\circ}$ y $8^{\circ}$ Año Básico, porque, a su edad, les resulta difícil discriminar entre muchos reactivos. Por lo tanto, para los sujetos de la investigación en desarrollo mencionada, se considera adecuado el uso de tres opciones de respuesta: Totalmente de acuerdo, Parcialmente de acuerdo y Totalmente en desacuerdo.

Para calcular los resultados, considerando que la escala de Likert puede contener ítems positivos y negativos, se procede de la siguiente manera:

a) A los ítems positivos se asigna las puntuaciones 3 y 1, para Totalmente de acuerdo y Totalmente en desacuerdo, respectivamente.

b) Para los ítems negativos, se invierten las puntuaciones, por lo tanto, se asignan los valores 1 y 3 para: Totalmente de acuerdo y Totalmente en desacuerdo, respectivamente.

\subsection{VALIDACIÓN DEL INSTRUMENTO}

En una investigación científica, los instrumentos de medición deben cumplir con condiciones mínimas de validez y confiabilidad. Según Ruiz (1998), la validez, en términos generales, se refiere al grado en que un instrumento realmente mide la variable que pretende medir. Al respecto señala que la validez es un concepto del cual puede obtenerse evidencias relacionadas con: el contenido, el criterio y el constructo. En este contexto, es preciso clarificar que, en la presente validación, no se busca la validez total, sino llevar a cabo un proceso de carácter exploratorio, que incluya la validez de contenido y la validez de constructo. En cuanto a la confiabilidad, Hernández et al. (1996:242) señalan: "La confiabilidad de un instrumento se refiere al grado en que su aplicación repetida al mismo sujeto u objeto, produce iguales resultados".

\subsubsection{Validez de contenido}

Es el grado en que la medición representa al concepto medido, en este caso, la metacognición, en términos de autoconocimiento y autorregulación. 
A partir de una amplia revisión bibliográfica sobre el constructo metacognición y su operacionalización, se construyó una Escala tipo Likert que estuvo compuesta por 120 ítems o proposiciones acerca de la actitud de los estudiantes hacia la metacognición con tres opciones de respuesta. El instrumento fue sometido a juicio de un panel de expertos, a fin de revisar los ítems según su pertinencia respecto del constructo metacognición, descartando aquéllos que no cumplieran con esta condición. Como producto del juicio de expertos, la escala quedó compuesta por un total de 100 ítems.

Con dicha escala se realizó una aplicación piloto a 102 estudiantes de $7^{\circ}$ y $8^{\circ}$ Año Básico de una Escuela de características similares, en cuanto a índice de vulnerabilidad y porcentaje de estudiantes de origen mapuche, a la Escuela en que se aplicaría; en definitiva, el instrumento en calidad de pretest y postest.

Realizada la aplicación piloto, se procedió a elaborar la base de datos con la información obtenida con el propósito de determinar la confiabilidad y validez de constructo del instrumento, mediante los procedimientos estadísticos Coeficiente Alfa de Cronbach y Análisis Factorial, respectivamente.

\subsubsection{Confiabilidad del instrumento}

Reforzando la definición de Hernández et al. (1996: 242) mencionada anteriormente, se puede entender "la confiabilidad como una propiedad de los instrumentos de medición consistente en que éstos, aplicados dos veces a los mismos sujetos (quienes no han cambiado en nada entre ambas aplicaciones), deben obtener el mismo valor. Esto implica definir la confiabilidad como la estabilidad de nuestras mediciones" (Canales, 2006: 101).

Con relación a la determinación de la confiabilidad del instrumento, a través del Coeficiente Alfa de Cronbach se descartaron todos aquellos ítems que disminuían la confiabilidad del modelo, quedando sólo aquellos que tenían un alfa igual o mayor a 0,7. En esta forma, la versión definitiva del instrumento quedó conformada por 33 ítems.

\subsubsection{Validez de constructo}

La validez de constructo que, según Carmines y Zeller (cit. en Hernández et al.,1996: 245), es la más importante, se refiere a mediciones consistentes de acuerdo a hipótesis que derivan de la teoría relacionada con los constructos a medir. Su determinación se realiza generalmente mediante el procedimiento estadístico denominado: análisis factorial o análisis de factores.

Para determinar la validez de constructo del presente instrumento, se realizó un análisis factorial, técnica que, basada en los datos, permitió descubrir su estructura subyacente, definiendo la configuración de las dimensiones que componen la escala. Mediante este análisis se agruparon los 33 ítems en 6 factores o dimensiones, a saber: Conocimiento ( 9 ítems), Control y Supervisión (5 ítems), Planificación (5 ítems), Experiencias (5 ítems), Evaluación (6 ítems) y Estrategias (3 ítems). 


\section{ANALISIS ESTADISTICOS}

\subsection{CONFIABILIDAD DEL INSTRUMENTO}

Para verificar la confiabilidad de los datos cuantitativos se utilizó el coeficiente Alfa de Cronbach, que requiere una sola administración del instrumento de medición y produce valores entre 0 y 1 .

El resultado de la aplicación del coeficiente Alfa de Cronbach, se puede observar en la Tabla $\mathrm{N}^{\circ} 1$.

\begin{tabular}{|c|c|}
\hline Alfa de Cronbach & $\mathrm{N}^{\circ}$ de Elementos \\
\hline 0,860 & 33 \\
\hline
\end{tabular}

Tabla 1

La interpretación del coeficiente de confiabilidad obtenido se realizó a partir de la Escala que se muestra a continuación.

\begin{tabular}{|c|c|}
\hline 0,81 a 1,00 & Muy alta \\
\hline 0,61 a 0,80 & Alta \\
\hline 0,40 a 0,60 & Moderada \\
\hline 0,21 a 0,40 & Baja \\
\hline 0,01 a 0,20 & Muy Baja \\
\hline
\end{tabular}

Tabla 2

A partir de la Tabla $\mathrm{N}^{\mathrm{o}} 2$, se puede inferir que el valor alfa $(0,860)$ obtenido implica un nivel de correlación promedio muy alto, por lo cual, se puede afirmar que el instrumento es confiable.

\subsection{VALIDEZ DE CONSTRUCTO DEL INSTRUMENTO}

Se obtuvo mediante el análisis de factores, método estadístico que permite determinar número y naturaleza de los constructos que subyacen a un grupo de mediciones.

Previo al análisis de factores propiamente tal, se realizaron operaciones tales como: adecuación muestral KMO, a fin de indagar si la muestra es apta para el análisis y prueba de esfericidad de Bartlett, a objeto de detectar la existencia de relaciones entre las variables.

En la Tabla $\mathrm{N}^{\mathrm{o}}$ 3, se observa que la medida de adecuación muestral KMO (KaiserMeyer- Olkin) indica que la muestra de investigación es óptima para realizar el análisis factorial. A su vez, la Prueba de esfericidad de Bartlett, la cual contrasta la hipótesis nula de que la matriz de correlaciones entre las variables consideradas constituye una matriz de identidad, de acuerdo al resultado obtenido (Chi cuadrado $=1398,882$; gl=528; 
p=,000), permite rechazar la hipótesis nula y justifica la realización del análisis factorial, al poner de manifiesto que existen relaciones relevantes entre las variables incluidas.

\begin{tabular}{|c|c|c|}
\hline \multicolumn{2}{|c|}{$\begin{array}{c}\text { Medida de adecuación muestral de Kaiser-Meyer- } \\
\text { Olkin }\end{array}$} & 0,752 \\
\hline $\begin{array}{c}\text { Prueba de esfericidad de } \\
\text { Bartlett }\end{array}$ & $\begin{array}{c}\text { Chi-cuadrado } \\
\text { aproximado }\end{array}$ & 1398,882 \\
\hline & gl & 528 \\
\cline { 2 - 3 } & Sig. & 0,000 \\
\hline
\end{tabular}

Tabla 3. KMO y Prueba de Bartlett

En la Tabla $\mathrm{N}^{\mathrm{o}}$ 4, que se muestra a continuación, se presentan los factores que componen el Instrumento sobre Metacognición y el porcentaje total de la varianza explicada. De acuerdo a los resultados, se observa que los factores explican el 55,24\% de la varianza total.

\begin{tabular}{|c|c|c|c|c|c|c|c|c|c|}
\hline \multirow{2}{*}{ Componente } & \multicolumn{3}{|c|}{ Autovalores iniciales } & \multicolumn{3}{|c|}{$\begin{array}{l}\text { Sumas de las Saturaciones al cuadrado } \\
\text { de la extracción }\end{array}$} & \multicolumn{3}{|c|}{$\begin{array}{c}\text { Sumas de las Saturaciones al cuadrado } \\
\text { de la rotación }\end{array}$} \\
\hline & Total & $\begin{array}{c}\% \text { de la } \\
\text { Varianza } \\
\end{array}$ & $\begin{array}{c}\% \% \\
\text { Acumulado } \\
\end{array}$ & Total & $\begin{array}{c}\% \text { de la } \\
\text { Varianza } \\
\end{array}$ & $\begin{array}{c}\% \% \\
\text { Acumulado } \\
\end{array}$ & Total & $\begin{array}{c}\% \text { de la } \\
\text { Varianza } \\
\end{array}$ & $\begin{array}{c}\% \\
\text { Acumulado }\end{array}$ \\
\hline 1 & 7,671 & 23,244 & 23,244 & 7,671 & 23,244 & 23,244 & 4,329 & 13,120 & 13,120 \\
\hline 2 & 3,014 & 9,133 & 32,377 & 3,014 & 9,133 & 32,377 & 3,653 & 11,068 & 24,188 \\
\hline 3 & 2,211 & 6,700 & 39,077 & 2,211 & 6,700 & 39,077 & 2,877 & 8,717 & 32,905 \\
\hline 4 & 1,884 & 5,710 & 44,787 & 1,884 & 5,710 & 44,787 & 2,728 & 8,268 & 41,173 \\
\hline 5 & 1,807 & 5,476 & 50,263 & 1,807 & 5,476 & 50,263 & 2,683 & 8,131 & 49,304 \\
\hline 6 & 1,642 & 4,975 & 55,239 & 1,642 & 4,975 & 55,239 & 1,958 & 5,935 & 55,239 \\
\hline 7 & 1,275 & 3,865 & 59,103 & & & & & & \\
\hline 8 & 1,148 & 3,478 & 62,581 & & & & & & \\
\hline 9 & 1,020 & 3,089 & 65,671 & & & & & & \\
\hline 10 & 0,946 & 2,868 & 68,539 & & & & & & \\
\hline 11 & 0,907 & 2,748 & 71,286 & & & & & & \\
\hline 12 & 0,878 & 2,662 & 73,948 & & & & & & \\
\hline 13 & 0,820 & 2,486 & 76,434 & & & & & & \\
\hline 14 & 0,755 & 2,289 & $\begin{array}{l}78,273 \\
\end{array}$ & & & & & & \\
\hline 15 & 0,703 & 2,132 & 80,854 & & & & & & \\
\hline 16 & 0,673 & 2,040 & 82,895 & & & & & & \\
\hline 17 & 0,585 & 1,772 & 84,666 & & & & & & \\
\hline 18 & 0,529 & 1,603 & 86,269 & & & & & & \\
\hline 19 & 0,507 & 1,535 & 87,805 & & & & & & \\
\hline 20 & 0,478 & 1,447 & 89,252 & & & & & & \\
\hline 21 & 0,446 & 1,352 & 90,604 & & & & & & \\
\hline 22 & 0,409 & 1,238 & 91,842 & & & & & & \\
\hline 23 & 0,404 & 1,223 & 93,065 & & & & & & \\
\hline 24 & 0,337 & 1,022 & 94,087 & & & & & & \\
\hline 25 & 0,327 & 0,990 & 95,078 & & & & & & \\
\hline 26 & 0,283 & 0,856 & 95,934 & & & & & & \\
\hline 27 & 0,258 & 0,781 & 96,715 & & & & & & \\
\hline 28 & 0,239 & 0,725 & 97,440 & & & & & & \\
\hline 29 & 0,214 & 0,647 & $\begin{array}{l}98,087 \\
\end{array}$ & & & & & & \\
\hline
\end{tabular}




\begin{tabular}{|l|l|l|l|l|l|l|l|l|l|}
\hline 30 & 0,185 & 0,560 & 98,647 & & & & & & \\
\hline 31 & 0,161 & 0,489 & 99,136 & & & & & & \\
\hline 32 & 0,151 & 0,459 & 99,595 & & & & & & \\
\hline 33 & 0,134 & 0,405 & 100,000 & & & & & & \\
\hline
\end{tabular}

Tabla 4. Varianza Total Explicada

En la Tabla $\mathrm{N}^{\mathrm{o}}$ 5, se presenta la matriz de componentes rotados, dando cuenta de la relación de cada una de las variables con los distintos factores ortogonales (independientes entre sí) generados a través de la rotación Varimax. Además, en la matriz, se presentan las cargas factoriales (pesos específicos de cada uno de los ítems en cada uno de los factores), pudiéndose determinar, de esta forma, los principales componentes del Instrumento Metacognitivo.

\begin{tabular}{|c|c|c|c|c|c|c|}
\hline & \multicolumn{6}{|c|}{ Componente } \\
\hline & Conoc. & Control & Planif. & Exper. & Eval. & Estrat. \\
\hline $\begin{array}{l}\text { 1. Es bueno descomponer un problema en problemas más pequeños } \\
\text { para resolverlo }\end{array}$ & 0,537 & & & & & \\
\hline $\begin{array}{l}\text { 2. Yo pienso en diversas maneras para resolver un problema y } \\
\text { luego escojo la mejor }\end{array}$ & 0,508 & & & & & \\
\hline 3. Yo imagino el problema para saber cómo resolverlo & 0,619 & & & & & \\
\hline 4. Yo decido lo que necesito hacer antes de comenzar una tarea & 0,570 & & & & & \\
\hline 5. Sé qué pasos debo seguir para resolver un problema & 0,642 & & & & & \\
\hline 6. Voy revisando los objetivos para saber si los estoy logrando & 0,600 & & & & & \\
\hline 7. Lo que aprendo me debe servir para comprender otras cosas & 0,687 & & & & & \\
\hline 8. Sé que aprendí cuando puedo explicar otros hechos & 0,662 & & & & & \\
\hline 9. Me puedo dar cuenta que no aprendí & 0,662 & & & & & \\
\hline $\begin{array}{l}\text { 10. Cuando voy a comenzar una tarea, me pregunto qué quiero } \\
\text { lograr }\end{array}$ & & 0,806 & & & & \\
\hline 11. Me propongo objetivos con cada tarea & & 0,724 & & & & \\
\hline 12. Me pregunto si lo estoy haciendo bien & & 0,542 & & & & \\
\hline $\begin{array}{l}\text { 13. Controlo el tiempo para saber si terminaré todo mi trabajo en } \\
\text { clases }\end{array}$ & & 0,612 & & & & \\
\hline $\begin{array}{l}\text { 14. Cuando termina la clase me pregunto si pude poner atención a } \\
\text { lo importante }\end{array}$ & & 0,647 & & & & \\
\hline 15 Para comprender más, leo y vuelvo a leer & & & 0,643 & & & \\
\hline 16. Yo necesito leer más lento cuando el texto es difícil & & & 0,756 & & & \\
\hline $\begin{array}{l}\text { 17. Yo creo que es bueno diseñar un plan antes de comenzar a } \\
\text { resolver una tarea }\end{array}$ & & & 0,679 & & & \\
\hline 18. Cuando no sé lo que significa una palabra la paso por alto & & & 0,511 & & & \\
\hline 19. Me siento más seguro (a) si planifico algo antes de hacerlo & & & 0,545 & & & \\
\hline 20. Para mí es difícil poner atención en clases & & & & 0,702 & & \\
\hline $\begin{array}{l}\text { 21. A mí me resulta más difícil que a mis compañeros aprender } \\
\text { matemáticas }\end{array}$ & & & & 0,684 & & \\
\hline $\begin{array}{l}\text { 22. Yo sé que mi memoria es frágil por lo que se me olvidan } \\
\text { algunas cosas }\end{array}$ & & & & 0,695 & & \\
\hline 23. Me distraigo con facilidad en clases & & & & 0,736 & & \\
\hline 24. Si aprendo de memoria se me olvida fácilmente & & & & 0,541 & & \\
\hline 25. Me molesta no entender en la clase & & & & & 0,691 & \\
\hline 26. Cuando tengo un error me gusta saber cuál es & & & & & 0,691 & \\
\hline 27. No me gusta quedar con dudas en una clase & & & & & 0,562 & \\
\hline 28. Cuando me saco una mala nota trato de mejorarla después & & & & & 0,509 & \\
\hline 29. Yo confío en lo que soy capaz de aprender & & & & & 0,548 & \\
\hline 30. Yo me preocupo de saber si aprendí & & & & & 0,516 & \\
\hline 31. Yo subrayo porque así aprendo más fácilmente & & & & & & 0,606 \\
\hline 32. A mí se me hace más fácil recordar subrayando & & & & & & 0,696 \\
\hline 33. Si no entiendo algo, prefiero preguntarle a mis compañeros & & & & & & 0,590 \\
\hline
\end{tabular}

Tabla 5. Matriz de componentes rotados ${ }^{\mathrm{a}}$

Método de extracción: Análisis de componentes principales

Método de rotación: Normalización Varimax con Kaiser

a. La rotación ha convergido en 9 iteraciones 
Los Factores extraídos mediante análisis factorial son los siguientes:

El Factor 1, "Conocimiento", está referido al conocimiento y creencias sobre las características de la persona según sus propias habilidades, motivaciones, recursos y estados personales; características de la persona en relación a otras personas y características de la persona como ser cognitivo. También se refiere al conocimiento de la persona sobre cómo la naturaleza y demanda de la tarea influye en su ejecución y aprendizaje. El factor se encuentra conformado por los ítems:

- Es bueno descomponer un problema en problemas más pequeños para resolverlo

- Yo pienso en diversas maneras para resolver un problema y luego escojo la mejor

- Yo imagino el problema para decidir cómo resolverlo

- Yo decido lo que necesito hacer antes de comenzar una tarea

- Sé qué pasos debo seguir para resolver un problema

- Voy revisando los objetivos para saber si los estoy logrando

- Lo que aprendo me debe servir para comprender otras cosas

- Sé que aprendí cuando puedo explicar otros hechos

- Me puedo dar cuenta que no aprendí

El Factor 2, "Control y supervisión", se refiere al componente procedimental que se establece desde que se inicia la ejecución de las acciones con el propósito de verificar y rectificar la estrategia empleada. Este factor está constituido por los ítems:

- Cuando voy a comenzar una tarea me pregunto qué quiero lograr

- Me propongo objetivos con cada tarea

- Me pregunto si lo estoy haciendo bien

- Controlo el tiempo para saber si terminaré todo mi trabajo en clases

- Cuando termina la clase me pregunto si pude poner atención a lo importante

-

El Factor 3, "Planificación", corresponde a la actividad previa a la ejecución de una tarea, es decir, acciones y estrategias a seguir. Está constituido por los ítems:

- Para comprender más leo y vuelvo a leer

- Yo necesito leer más lento cuando el texto es difícil

- Yo creo que es bueno diseñar un plan antes de comenzar a resolver una tarea

- Cuando no sé lo que significa una palabra la paso por alto

- Me siento más seguro(a) si planifico algo antes de hacerlo

El Factor 4, "Experiencias", se refiere al pensamiento, emociones, sensaciones o sentimientos que acompañan la actividad cognitiva de una persona y que pueden influir en el progreso hacia la meta. Está constituido por los ítems:

- Para mí es difícil poner atención en clases

- A mí me resulta más difícil que a mis compañeros aprender matemáticas

- Yo sé que mi memoria es frágil por lo que se me olvidan algunas cosas

- Me distraigo con facilidad en clases 
- Si aprendo de memoria se me olvida fácilmente

El Factor 5, "Evaluación", se refiere a la acción de contrastar los resultados con los propósitos definidos previamente. Está constituido por los ítems:

- Me molesta no entender en la clase

- Cuando tengo un error me gusta saber cuál es

- No me gusta quedar con dudas en una clase

- Cuando me saco una mala nota trato de mejorarla después

- Yo confío en lo que soy capaz de aprender

- Yo me preocupo de saber si aprendí

-

El Factor 6, "Estrategias", se refiere al empleo de diferentes acciones destinadas a hacer progresar la actividad cognitiva hacia la meta. Este factor está constituido por los ítems:

- Yo subrayo porque así aprendo más fácilmente

- A mí se me hace más fácil recordar subrayando

- Si no entiendo algo prefiero preguntarle a mis compañeros

\section{INSTRUMENTO DEFINITIVO}

El instrumento definitivo, que se presenta a continuación, consta de 33 ítems con 3 alternativas de respuesta: Totalmente de acuerdo, Parcialmente de acuerdo, Totalmente en desacuerdo, para ser administrado de forma individual en un tiempo promedio de 20 minutos.

\section{INSTRUMENTO DE METACOGNICIÓN}

Estimado (a) estudiante:

Con el objeto de apoyarte para que mejores tus aprendizajes, a continuación te presentamos un conjunto de afirmaciones que te solicitamos encarecidamente respondas completo en la forma más honesta posible.

Por favor, marca con una X la respuesta que más se aproxime a lo que tú piensas o haces.

\begin{tabular}{|c|c|c|c|}
\hline & $\begin{array}{c}\text { Totalmente de } \\
\text { acuerdo }\end{array}$ & $\begin{array}{c}\text { Parcialmente } \\
\text { de acuerdo }\end{array}$ & $\begin{array}{c}\text { Totalmente } \\
\text { en } \\
\text { desacuerdo }\end{array}$ \\
\hline $\begin{array}{c}\text { 1. Es bueno descomponer un } \\
\text { problema en problemas más } \\
\text { pequeños para resolverlo }\end{array}$ & & & \\
\hline & & & \\
\hline
\end{tabular}




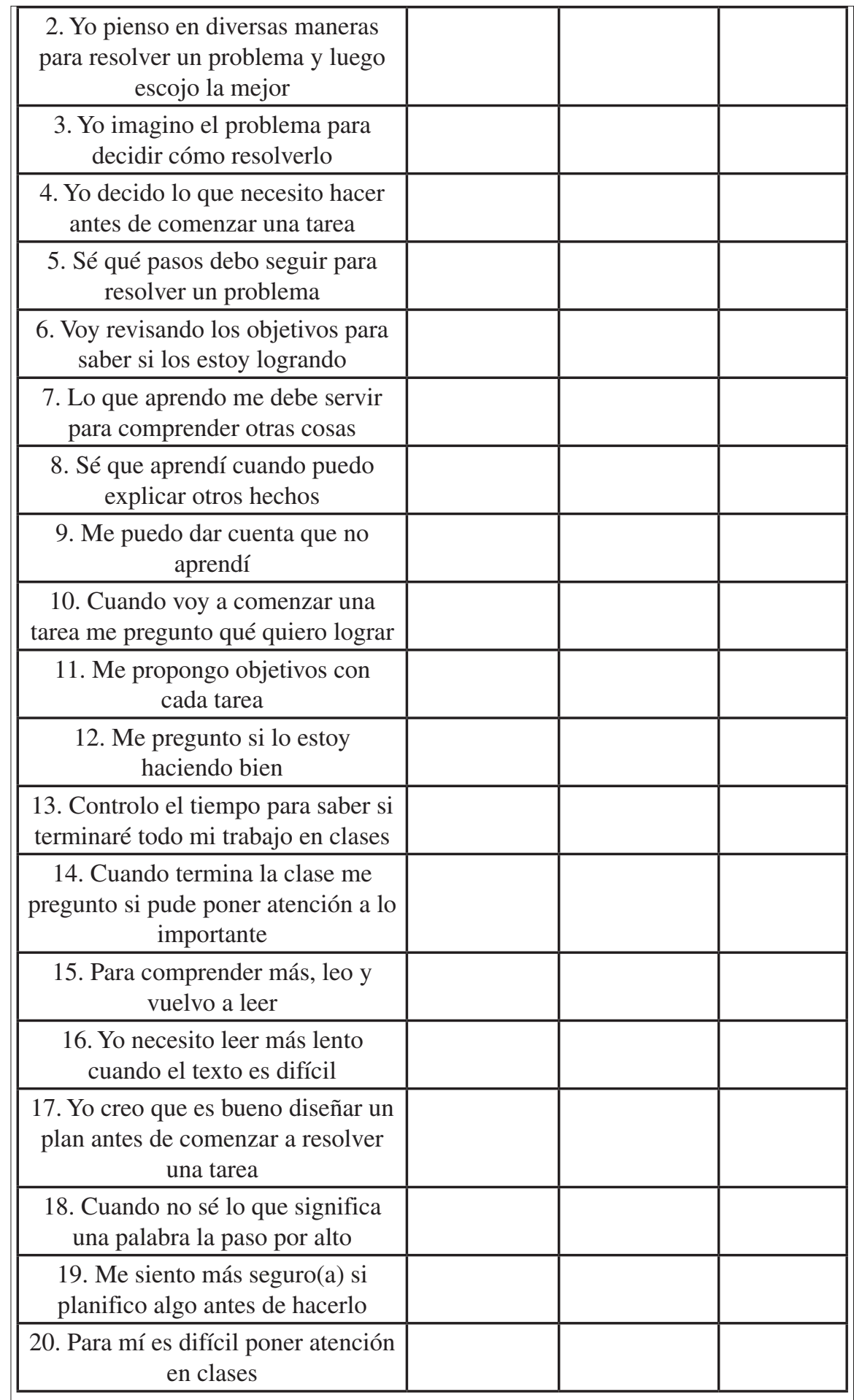




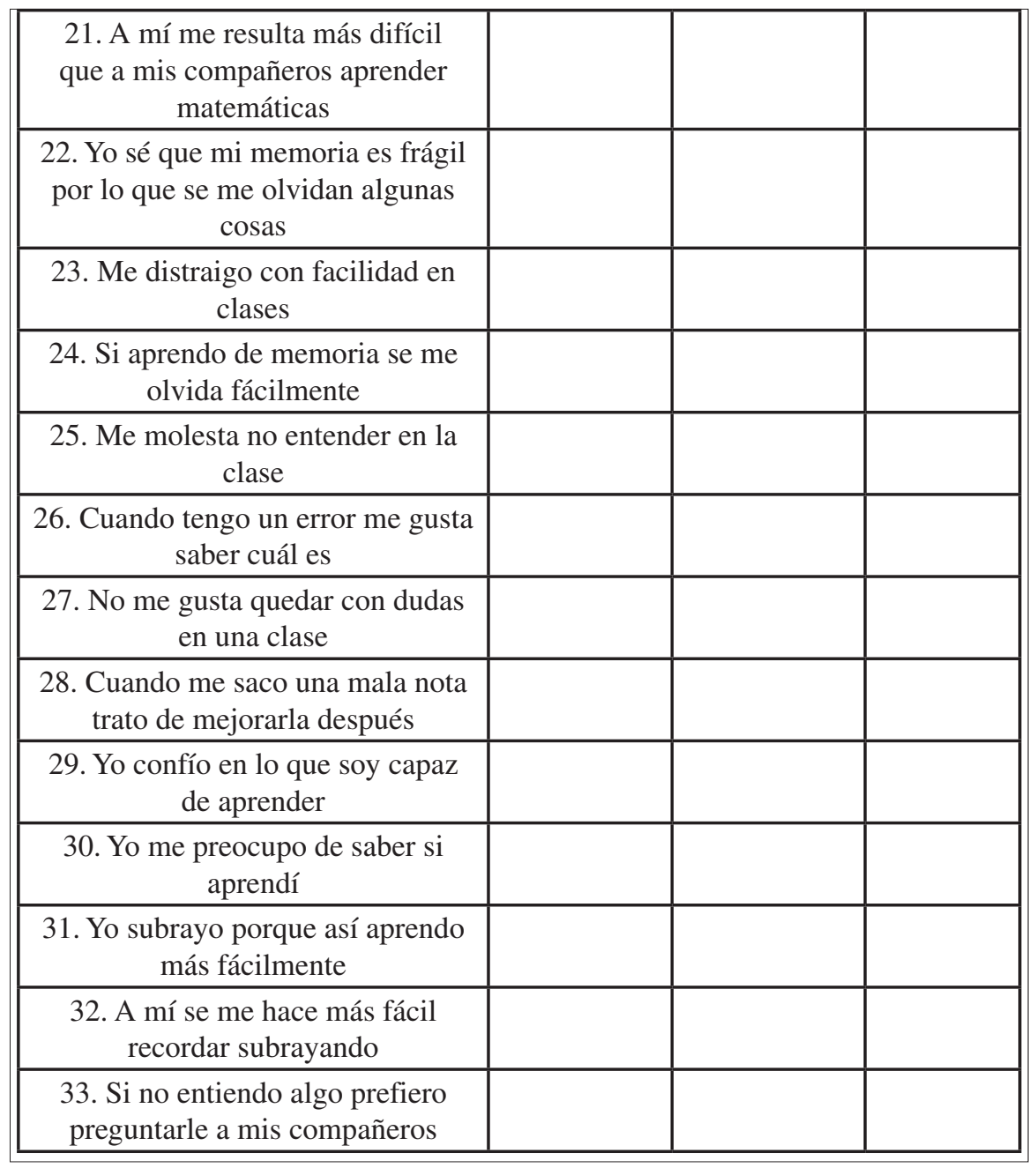

Instrumento de Metacognición

\section{PALABRAS FINALES}

A pesar de los esfuerzos realizados para medir la metacognición en niños y jóvenes, en Chile, a excepción del equipo integrado por Peronard et al. (2000) que ha validado un instrumento para medir sólo metacomprensión lectora en estudiantes de Educación General Básica y Media de la Quinta Región, aún no se cuenta en nuestro país, con instrumentos válidos y confiables que permitan medir el constructo metacognición en la totalidad de sus dimensiones.

En este contexto, los instrumentos que miden metacognición, de los cuales da cuenta la literatura especializada, deben adaptarse para su aplicación a estudiantes de Segundo Ciclo de Educación General Básica Chilena, en cuanto a la naturaleza y número de reactivos como asimismo, a la denominación y número de opciones de respuesta. 
Surge así la necesidad de diseñar y validar un instrumento para medir metacognición -como un camino que conduce a la autonomía del aprendizaje que responda a las características socioculturales de los estudiantes, de modo que los resultados de dicha validación favorezcan la generación de experiencias pedagógicas significativas a partir de un contexto local.

En el presente artículo, se ha hecho referencia a la validación de un instrumento tipo escala de Likert, con carácter exploratorio, como una primera aproximación a la medición de la metacognición en términos de conocimiento y autorregulación, dirigido a estudiantes de $7^{\circ}$ y $8^{\circ}$ Año Básico de Escuelas Municipalizadas de la Región de La Araucanía.

A partir de los análisis estadísticos realizados, se puede afirmar que la Escala Metacognitiva a cuya validación se hace referencia en este artículo, presenta las siguientes propiedades psicométricas:

a. Confiabilidad. Se determinó mediante el coeficiente Alfa de Cronbach obteniéndose un valor de 0.860 , el cual puede considerarse muy alto de acuerdo a la escala de valores propuesta por Ruiz (1998). Como esta medida de confiabilidad se basa en la consistencia interna del instrumento, se puede afirmar que los ítems miden la misma variable, es decir, todos se refieren al constructo metacognición.

b. Validez de contenido. Se determinó a través de un panel de expertos, que filtró los ítems dejando sólo los pertinentes al foco temático del instrumento: la metacognición.

c. Validez de constructo: Se determinó mediante Análisis Factorial. Los resultados obtenidos a partir de este análisis señalan la existencia de 6 factores: conocimiento, control, supervisión, planificación, experiencias, evaluación y estrategias, correspondiendo éstas a cada uno de los elementos componentes del proceso metacognitivo.

Por último, las autoras del presente artículo estiman que, si bien se ha logrado realizar un estudio exploratorio de validación de un instrumento para medir metacognición en estudiantes de Educación General Básica Chilena sobre la base de: validez de contenido, análisis de confiabilidad y validez de constructo, sería recomendable continuar dicho proceso a fin de lograr validez total del instrumento, determinando: validez de criterio, de contenido y de constructo. Por lo tanto, para el presente instrumento, queda pendiente la determinación de validez de criterio, es decir, la correlación entre la medición realizada y un criterio externo relacionado con el constructo metacognición.

\section{REFERENCIAS BIBLIOGRÁFICAS}

Argüelles, D. (2007). Estrategias para promover procesos de aprendizaje autónomo. Bogotá: Alfaomega Colombiana S.A.

Bermeosolo, J. (2005). Cómo aprenden los seres humanos. Mecanismos psicológicos del aprendizaje. Santiago. Chile: Universidad Católica de Chile.

Canales, M. (2006). Metodología de investigación social. Introducción al oficio. Chile: LOM Ediciones.

Carretero, M. (2001). Metacognición y educación. Buenos Aires: Aique.

Colas, M. Y Buendia, L. (1998). Investigación Educativa. Sevilla, España: Ediciones Alfar.

Hernandez, R. Fernández, C. y Baptista, P. (1996). Metodología de la investigación. Editorial McGraw-Hill.

Jiménez, V. (2004). Metacognición y Comprensión de la lectura: evaluación de los componentes estratégicos (Procesos y variables) mediante la elaboración de una escala de conciencia lectora 
(ESCOLA). Recuperado 12 enero 2011. http://www.unne.edu.ar/institucional/documentos/ lecturayescritura08/jimenez.pdf

Mateos, M. (2001). Metacognición y educación. Argentina: Aique Grupo Editorial.

Monereo, C., Badia, A., Baixeras, M., Boadas, E., Castello, M., Guevara, I., Miquel, E., Monte, M. Y Sebastiana, E. (2001). Ser estratégico y autónomo aprendiendo. Unidades didácticas de enseñanza estratégica. Barcelona. España: Graó.

Osses, S. (2008). Proyecto Fondecyt Regular $N^{\circ}$ 1070256: Hacia un aprendizaje autónomo en el ámbito científico. Inserción de la dimensión metacognitiva en el proceso educativo. Primer Informe de Avance.

Osses, S. (2011). Proyecto Fondecyt 1100378. "Hacia un aprendizaje de calidad en la educación científica. Estrategias didácticas para el desarrollo de capacidades y valores. Primer Informe de Avance.

Peronard, M., Crespo, N., Velásquez, M. (2000). La evaluación del conocimiento metacomprensivo en alumnos de educación básica. Revista Signos, vol. XXXIII, n. 47, 168-180.

Ruiz, C. (1998). Instrumentos de Investigación Educativa. Venezuela: Barquisimeto, CIDEG.

Soto, C. (2002). Metacognición, cambio conceptual y Enseñanza de las Ciencias. Bogotá: Didáctica Magisterio. 